\title{
Divergence in a master variator generates distinct phenotypes and transcriptional responses
}

\author{
Jennifer E.G. Gallagher, ${ }^{1,2}$ Wei Zheng, ${ }^{3}$ Xiaoqing Rong, ${ }^{2}$ Noraliz Miranda, ${ }^{4}$ Zhixiang Lin, ${ }^{5}$ \\ Barbara Dunn, ${ }^{1}$ Hongyu Zhao, ${ }^{6,7,8}$ and Michael P. Snyder ${ }^{1,9}$ \\ ${ }^{1}$ Department of Genetics, Stanford University, Stanford, California 94305, USA; ${ }^{2}$ Department of Biology, West Virginia \\ University, Morgantown, West Virginia 26506, USA; ${ }^{3}$ Keck Biostatistics Resources, Yale University, New Haven, Connecticut \\ 06510, USA; ${ }^{4}$ University of Puerto Rico, Aguadilla, Puerto Rico 00604; ${ }^{5}$ Biological and Biomedical Sciences Graduate Program, \\ Yale University, New Haven, Connecticut 06520, USA; ${ }^{6}$ Program in Computational Biology and Bioinformatics, Yale University \\ School of Medicine, New Haven, Connecticut 06520, USA; 7 Department of Epidemiology and Public Health, Yale University \\ School of Medicine, New Haven, Connecticut 06520, USA; ${ }^{8}$ Department of Genetics, Yale University School of Medicine, New \\ Haven, Connecticut 06520, USA
}

Genetic basis of phenotypic differences in individuals is an important area in biology and personalized medicine. Analysis of divergent Saccharomyces cerevisiae strains grown under different conditions revealed extensive variation in response to both drugs (e.g., 4-nitroquinoline 1-oxide [4NQO]) and different carbon sources. Differences in 4NQO resistance were due to amino acid variation in the transcription factor Yrr1. Yrr1 ${ }^{\text {YJM789 }}$ conferred 4NQO resistance but caused slower growth on glycerol, and vice versa with Yrr1 ${ }^{\text {S96 }}$, indicating that alleles of Yrr1 confer distinct phenotypes. The binding targets of Yrr1 alleles from diverse yeast strains varied considerably among different strains grown under the same conditions as well as for the same strain under different conditions, indicating that distinct molecular programs are conferred by the different Yrr1 alleles. Our results demonstrate that genetic variations in one important control gene (YRR1), lead to distinct regulatory programs and phenotypes in individuals. We term these polymorphic control genes "master variators."

[Keywords: 4NQO; S. cerevisiae; genetic variation; polymorphism; transcription factor]

Supplemental material is available for this article.

Received August 18, 2013; revised version accepted January 13, 2014.

How genetic variation produces different phenotypes and the ability to adapt to distinct environmental challenges is an important question in biology. Polymorphisms in coding regions and regulatory elements have been shown to associate with changes in individual morphological phenotypes of multicellular organisms and survival characteristics in microorganisms (Rebeiz et al. 2009; Chan et al. 2010; Gerke et al. 2010; Davidson 2011). However, the extent to which variations in a single locus can generate entirely distinct regulatory programs and phenotypes is not well understood.

Alterations in gene regulatory components have been shown to vary in a range of organisms and can lead to distinct phenotypes. Loss of activity of transcription factors (TFs) can lead to phenotypic differences; for example, the loss of Pitx1 expression in stickleback fish

${ }^{9}$ Corresponding author

E-mail mpsnyder@stanford.edu

Article is online at http://www.genesdev.org/cgi/doi/10.1101/gad.

228940.113.Freely available online through the Genes \& Development

Open Access option. leads to different body morphologies (Chan et al. 2010; Infante et al. 2013), while variation in some TFs (e.g., Ime1, Rme1, and Rsf1) can alter sporulation efficiency in different Saccharomyces cerevisiae strains (Gerke et al. 2009). Additionally, differences in gene expression and TF-binding sites have been shown to vary considerably between closely related species and individuals, although how these differences affect phenotypes is often unknown (Borneman et al. 2006; Zheng et al. 2010). Although we know that phenotypic changes can result from total loss of activity of TFs, it remains unclear whether allelic variations in a single TF can mediate broad differences in molecular responses and complex phenotypes through network rewiring. Such changes would be expected to have important implications in the rapid evolution of complex phenotypes and in allowing the adaptation of organisms to new environments.

(C) 2014 Gallagher et al. This article, published in Genes \& Development, is available under a Creative Commons License (AttributionNonCommercial 3.0 Unported), as described at http://creativecommons. org/licenses/by-nc/3.0/. 
To better understand how phenotypes vary and evolve among different individuals within a population or among populations within a species, we analyzed different yeast strains to find proteins that can regulate the phenotypic response to the environment. Natural yeast strains are more genetically diverse than humans (Wei et al. 2007) and have been shown to vary considerably from laboratory strains in their response to different environmental conditions such as growth on distinct carbon sources and incubation in the presence of compounds and drugs (Liti et al. 2009). The loci mediating some phenotypic differences among different yeasts have been mapped (Gerke et al. 2009; Ehrenreich et al. 2010; Zheng et al. 2010), although the number of genes that have actually been identified is very limited.

We mapped the genetic basis of resistance/sensitivity to 4-nitroquinoline 1-oxide (4NQO), a bulky alkylating agent, in two yeast strains with very different responses to this drug and identified a gene, YRR1, whose variation strongly contributed to this phenotypic difference. Enhanced fitness in one condition resulted in diminished fitness in another. When cells were able to grow well in the presence of $4 \mathrm{NQO}$, they then grew poorly in glycerol. The phenotypic difference was largely associated with changes in a single amino acid in Yrrl that was a potential site of phosphorylation. Importantly, Yrr1 bound different gene targets in different strain backgrounds, indicating that variation in a single TF can rewire transcriptional networks. Thus, natural polymorphisms in a single regulatory gene can cause broad molecular changes that result in distinct phenotypes in individuals, a phenomenon that has not been directly observed previously. To distinguish this kind of protein sequence variation from deleterious mutations, we term such genes whose natural polymorphisms that cause broad molecular changes master variators.

\section{Results}

\section{Different growth phenotypes among different yeast strains}

To explore the role of natural genetic variation in causing phenotypic differences, we first analyzed the growth of five haploid $S$. cerevisiae strains from diverse genetic and ecological backgrounds under a variety of environmental conditions. The strains examined were a common laboratory strain, S96 (derived from S288c background); two clinical isolates, YJM789 and YJM339 (McCusker et al. 1994); and two vineyard isolates, AWRI1631 (Borneman et al. 2008) and RM11 (Mortimer et al. 1994; Brem et al. 2002). The phenotypes analyzed included relative growth in different carbon sources (glucose, ethanol, galactose, and glycerol) and growth in the presence of different drugs (4NQO, fluconazole, hydroxyurea, and nystatin) (Fig. 1A). Fluconazole and nystatin both affect sterol metabolism, and YJM789 sensitivity to these chemicals has been recently linked to polymorphisms in the multidrug transporter Pdr5 (Guan et al. 2010). The genetic variation of response to hydroxyurea remains unknown. The five strains

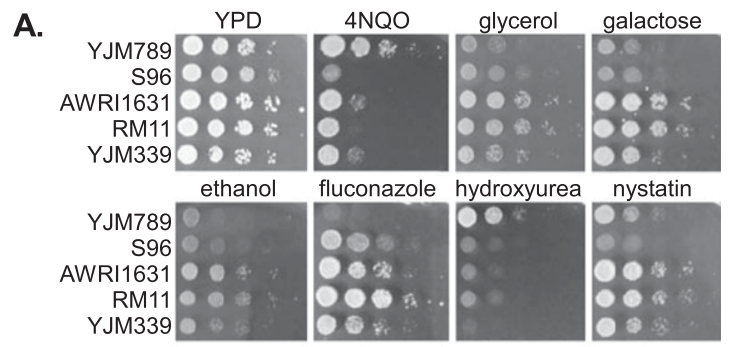

C.

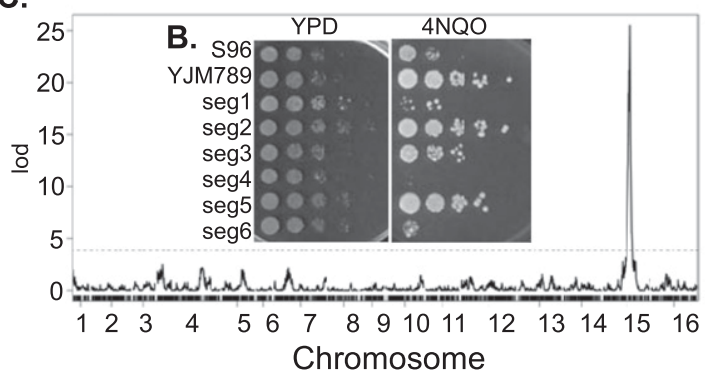

Figure 1. Extensive variation in yeast growth under different conditions and mapping of 4 NQO response. (A) Tenfold serial dilution of various strains of yeast spotted onto plates containing YPD, YPD + $0.2 \mu \mathrm{g} / \mathrm{mL} 4 \mathrm{NQO}, \mathrm{YP}+3 \%$ glycerol, YP + $2 \%$ galactose, $2 \%$ ethanol, YPD $+5 \mu \mathrm{g} / \mathrm{mL}$ fluconazole, YPD $+200 \mathrm{mM}$ hydroxyurea, and YPD $+2 \mu \mathrm{g} / \mathrm{mL}$ nystatin and grown for $1-3 \mathrm{~d}$ at $28^{\circ} \mathrm{C}$ before being photographed. $(B)$ Tenfold serial dilution of S96, YJM789, and six segregants spotted onto plates containing YPD and $0.2 \mu \mathrm{g} / \mathrm{mL} 4 \mathrm{NQO}$ and grown for $1-3 \mathrm{~d}$ at $28^{\circ} \mathrm{C}$ before being photographed. $(C)$ Genome-wide analysis study identified a region on chromosome 15 with a LOD score of 25.50 for the 95\% Bayes credible interval 639,300-640,218.

exhibited distinct growth characteristics under each of the different conditions (Fig. 1A), and each strain had a unique pattern of phenotypes. For example, relative to other yeasts, RM11 grew well under fluconazole, and YJM789 strains grew well in the presence of 4NQO; S96 cells grew slightly better on glycerol relative to YJM789 cells. Because the 4NQO resistance/sensitivity phenotype showed the most variation between the strains, we chose to map the genetic basis of this phenotype using the YJM789 and S96 strains, which exhibited the most extreme phenotypes.

To map the loci responsible for the 4NQO response variation, we used two previously sequenced yeast strains (YJM789 and S96) and a large collection of 125 haploid segregants that resulted from their cross (Wei et al. 2007; Mancera et al. 2008); the genetic content of each of these segregants was mapped at high resolution with highdensity microarrays. As shown in Figure 1B and Supplemental Figure S1, variation in the $4 \mathrm{NQO}$ response among the different segregants was observed. The response of segregants was scored and used as a quantitative trait for linkage analysis between the individual trait and genetic markers (see the Supplemental Material; Zheng et al. 2010). A strong peak associated with 4 NQO response was mapped to the right arm of chromosome 15 with a peak logarithm (base 10) of odds (LOD) score of 25.5 (Fig. 1C). Four other identified genomic locations, on chromosomes 
$1,4,5$, and 7 , had LOD scores outside the $95 \%$ confidence interval (Supplemental Table 1) and thus were not investigated further.

Yrr1 mediates distinct responses to 4 NQO and growth on glycerol in different yeasts

Because of the large number of segregants analyzed, the region associated with 4NQO response could be mapped to a very narrow interval (Fig. 2A). Within this region was $Y R R 1$, a gene encoding a TF. A strain containing a hypermorphic allele of YRR1 was previously found to be resistant to 4NQO (Cui et al. 1998), but the variation of YRR1 among different yeasts and its potential role in conferring differential 4NQO resistance had not been studied previously. To determine whether $Y R R 1$ was responsible for mediating variation in the 4NQO response, we swapped the YRR1 alleles (termed YRR $1^{Y J M 789}$ and $Y R R 1^{\mathrm{S} 96}$ ) between the two genetic backgrounds. $Y R R 1^{\mathrm{S} 96}$ and YRR $1^{\mathrm{YJM} 789}$ alleles on centromeric plasmids were transformed into both wild-type and yrr1s mutant strains. Importantly, when either the S96 wild-type strain or its yrr1s derivative was expressing YRR1 $1^{\mathrm{YIM} 789}$, it showed a strong increase in $4 \mathrm{NQO}$ resistance compared with S96 carrying an empty plasmid (Fig. 2B, rows 2, 7). As expected, introduction of YRR $1^{\mathrm{YJM} 789}$ into YJM789 yrr1s cells restored 4NQO resistance (Fig. 2B, rows 1, 4 vs. control S96 rows 2, 8; Supplemental Fig. S2). Wildtype YJM789 cells containing the YRR $1^{\text {YJM789 allele were }}$ 4NQO-resistant, also as expected. YJM789 yrr1s strains containing the $Y R R 1^{\mathrm{S} 96}$ allele as well as cells lacking any plasmid had intermediate sensitivity to 4NQO, indicating that YRR1 is the major contributor to $4 \mathrm{NQO}$ resistance in this strain (Fig. 2A,B). We can also conclude that the YRR $1^{\text {YJM789 }}$ allele is dominant over the YRR $1^{\mathrm{S} 96}$ allele. The strong differences in growth observed on plates were also evident using quantitative growth assays of cells grown in liquid cultures containing 4NQO (Fig. 2C).

In our initial screens, we found that S96 cells grew better on glycerol medium than YJM789 cells. We therefore examined whether YRR1 was involved in this phenotypic difference. Remarkably, we found that YJM789 strains (both wild type and yrr1s) exhibited a modest, but reproducible, increased growth on plates containing glycerol medium with expression of the $Y R R 1^{\mathrm{S} 96}$ allele. Interestingly, S96 cells containing the YRR $1^{Y J M 789}$ allele exhibited slightly slower growth on glycerol plates. The results found on the plates were also reflected in the quantitative cell culture assays (Fig. 2C); YJM789 cells with the $Y R R 1^{S 96}$ allele grew $16 \%$ faster on glycerol than wild-type YJM789 cells, while the S96 cells with YRR $1^{\text {YJM789 }}$ had a $17 \%$ slower growth rate on glycerol than wild-type S96 cells. Overall, these results indicate that the YRR $1^{Y I M 789}$ allele provided increased resistance to $4 \mathrm{NQO}$ in the S96 background but reduced growth on glycerol in either the YJM789 or S96 background. In contrast, the $Y R R 1^{\mathrm{S} 96}$ allele provided little to no resistance to $4 \mathrm{NQO}$ but led to increased growth on glycerol in either strain background. These results indicate that different YRR1 alleles confer distinct phenotypes and
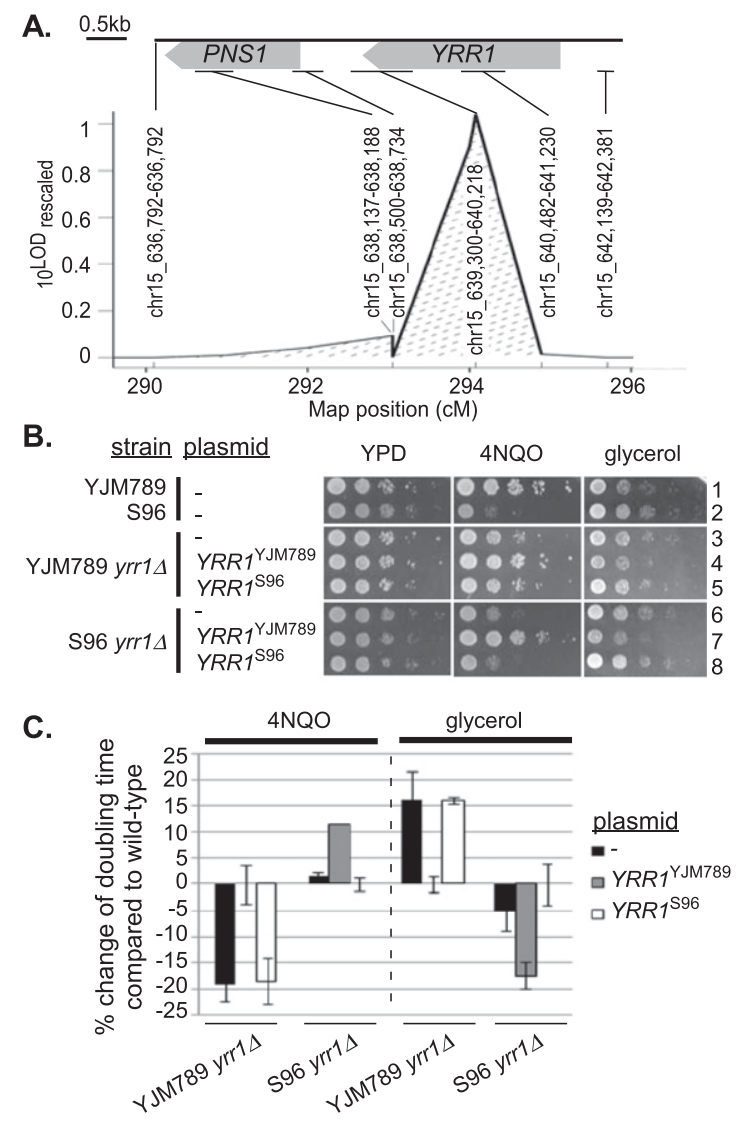

Figure 2. Different alleles of $Y R R 1$ are responsible for $4 \mathrm{NQO}$ resistance and rate of growth on glycerol. (A) Ninety-five percent Bayes credible interval for the QTL region on chromosome 15 (coordinates $636,762-645,935$ ) of the 4NQO data. The LOD curve was calculated by standard interval mapping using EM algorithm, and the $Y$-axis is $10^{\wedge} \mathrm{LOD}$ rescaled to make the area under the curve within $95 \%$ interval (shaded area) equal 1. Associated marker names are also shown. Note that two markers fall on the same map position, although their physical locations are different (chromosome 15: 638,137-638,188, and chr15: $638,500-638,734)$. Genes are drawn to physical scale, and the interval for each genetic marker is noted by lines between the physical map in kilobases and map position in centimorgans. Within this interval are the following genes: PNS1 (coordinates: 638,559-636,940) and YRR1 (coordinates: 641,993-639,561). (B) Tenfold serial dilution of wild-type strains YJM789 and S96 and yrr1s mutants carrying an empty plasmid (pGS35, a KAN ${ }^{\mathrm{R}}$ plasmid), pYRR $1^{\mathrm{YJM} 789}$, or $\mathrm{p} Y R R 1^{\mathrm{S} 96}$ encoding the respective alleles of $Y R R 1$ expressed from the endogenous promoters. Strains were growth on YPD, YPD $+0.25 \mu \mathrm{g} / \mathrm{mL} 4 \mathrm{NQO}$, and glycerol with $200 \mu \mathrm{g} / \mathrm{mL} \mathrm{G} 418$ and grown for $1-3 \mathrm{~d}$ at $28^{\circ} \mathrm{C}$ before being photographed. $(C)$ For cells grown in culture, the percentage

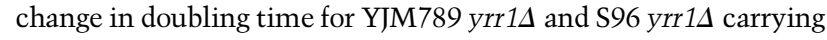
an empty plasmid or the exchanged allele as compared with YJM789 yrr1s and S96 yrr1s carrying their respective alleles.

that the YRR1 variations that improve growth in the presence of 4NQO cause reduced growth in glycerol.

\section{Differences in coding sequences confer phenotypic differences}

Inspection of the predicted protein sequences of Yrr1 reveals that they contain multiple amino acid polymor- 
phisms among the YJM789, S96, AWRI1631, RM11, and YJM339 strains. Four out of the five polymorphisms within the coding region were nonconservative changes and were located near the $\mathrm{C}$ terminus, and all were outside the putative $\mathrm{Zn}$ finger DNA-binding, coiled-coil, and activation domains (Fig. 3A). There were also four polymorphisms seen in the promoter regions of YRR1 $1^{Y I M 789}$ and $Y R R 1^{S 96}$. Two polymorphisms downstream from the YRR1 gene are not transcribed (Xu et al. 2009) and were not further studied. To determine whether the different
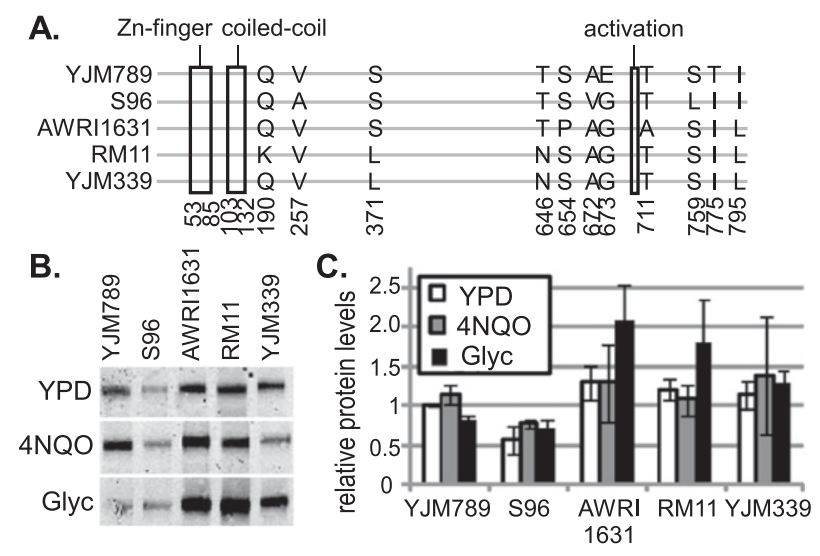

D.
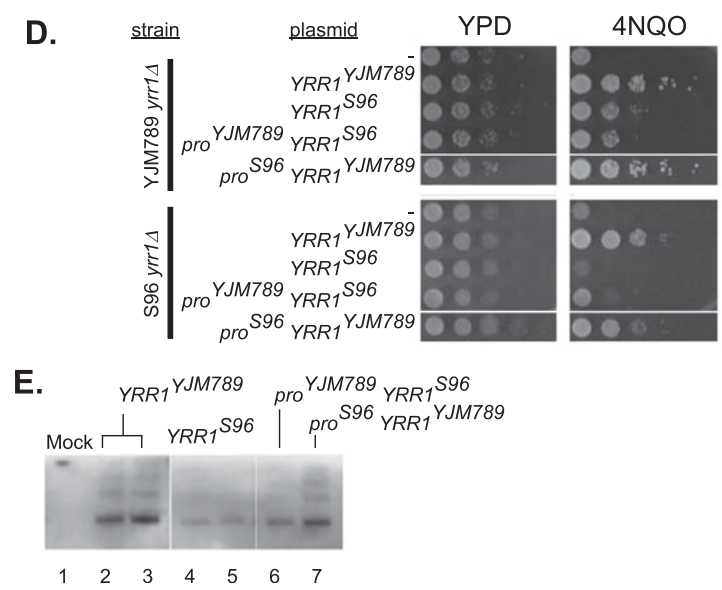

Figure 3. Yrrl contains polymorphisms in coding regions that are responsible for most of the variation in 4NQO response. $(A)$ Sequence alignment of Yrrl from five yeast strains. Polymorphisms are indicated with $\mathrm{Zn}$ finger, coiled-coil, and activation domain boxed. $(B)$ Immunoblot analysis of Yrr1-Myc immunoprecipitated from lysates from YJM789, S96, AWRI1631, RM11, and YJM339 strains grown in YPD, 4NQO for $2.5 \mathrm{~h}$, and glycerol for $8.5 \mathrm{~h} .(C)$ The levels of Yrrl immunoprecipitated via the $13 x M y c$ tag from yeast were quantitated relative to Yrr1 from YJM789 grown in YPD. An average of two to three experiments is shown with standard deviations. $(D)$ Tenfold serial dilution of S96 yrr14 and YJM789 yrr14 carrying YRR1 with promoters exchanged on plasmid pGS35; strains were incubated on YPD and YPD $+0.25 \mu \mathrm{g} / \mathrm{mL} 4 \mathrm{NQO}$ plates for $1-2 \mathrm{~d}$ at $28^{\circ} \mathrm{C}$ before being photographed. (E) Immunoblot of $\mathrm{N}$-terminally Myc tagged Yrrl expressed from a plasmid with allelic promoter or transallelic promoter and immunoprecipitated from cells grown in YPD. Cellular extract from cells carrying Yrrl ${ }^{\text {YIM789 }}$-Myc tag was incubated with protein A-conjugated agarose beads to control for nonspecific interactions and run in lane 1, labeled Mock. phenotypes among strains were due to differences in the YRR1 promoter or in their coding sequences, several tests were performed.

We first examined Yrr1 protein levels in all five backgrounds using immunoblot analyses (Fig. 3B; see also the legend). Yrr1 was evident in all five strains (Fig. 3B), although the levels varied modestly between strain and condition. For cells grown on YPD and other conditions, the levels of Yrrl from all strains were compared with Yrr1 ${ }^{\text {YJM789 }}$ grown in YPD. The level of $\mathrm{Yrr}^{\mathrm{S} 96}$ protein was $0.5 \pm 0.2$-fold (YPD) or less (4NQO and glycerol) than that of Yrr1 ${ }^{\text {YJM789 }}$ protein (average of three experiments) (Fig. 3C). There was also a similar lower level of S96 YRR1 mRNA (0.60) compared with that of YJM789 using DNA microarray analyses (data not shown). However, the level of Yrrl did not correlate to the rate of growth in any condition or strain. Therefore, to directly determine whether differences in expression of YRR1 itself or coding polymorphisms alter the 4NQO response, we exchanged the promoter regions of YRR1 from YJM789 and S96 backgrounds. The promoters from the two alleles of YRR1 were cloned in front of the other YRR1 allele on a plasmid and expressed in both YJM789 yrr14 and S96 yrr14. In S96 cells, we detected only a very small increase of 4NQO resistance when $Y R R 1^{596}$ expression was driven by the $Y R R 1^{Y I M 789}$ promoter (Fig. 3D). Expression of the YRR $1^{\text {YJM789 }}$ allele under the control of the YRR $1^{\mathrm{S} 96}$ promoter conferred strong $4 \mathrm{NQO}$ resistance and was similar to YRR1 $1^{Y J M 789}$ under its endogenous promoter (Fig. 3D). Protein levels were also assessed by immunoblotting of Myc-tagged Yrrl proteins immunoprecipitated from yeast with both YRR1 alleles driven from each's endogenous promoter or the promoter from the other allele. There was only a slight increase of $\mathrm{Yrr}^{\mathrm{S} 96}$ protein driven from the $Y R R 1^{Y J M 789}$ promoter and a slight decrease of Yrr1 ${ }^{\mathrm{YJM} 789}$ protein driven from the $Y R R 1^{S 96}$ promoter (Fig. 3E). Therefore, although YRR1 expression levels may contribute slightly to the different phenotypes, the polymorphisms in the coding region appear to be primarily responsible for alterations in Yrrl.

\section{Specific variable amino acids in Yrr1 regulate 4NQO response}

Because all of the variation among the alleles of YRR1 was outside the DNA-binding domain, the variation in Yrrl function appeared to due to differences that lay in other domains of the protein. Longer exposures of the immunoblots revealed higher-molecular-weight proteins that were detected with anti-Myc (Fig. 3E). Mock immunoprecipitations of Myc-tagged Yrrl showed no nonspecific interactions (Fig. 3E, lane 1). To investigate the nature of the slower-migrating bands of Yrrl, we treated lysates with phosphatase (calf intestinal phosphatase [CIP]) and immunoprecipitated Yrrl. The band with the same migrating pattern as the predicted Yrr1-Myc protein showed increased mobility, and some of the slower bands also shifted down to the faster-migrating band. The fastestmigrating band of Yrrl also showed a modest increase when immunoprecipitated Yrr1 ${ }^{\text {YJM789 }}$ was treated with 
CIP (Fig. 4A). At least one of the post-translational modifications of Yrr1 was sensitive to dephosphorylation treatment.

We compared the five coding polymorphisms between Yrrl $^{\text {YJM789 }}$ and Yrr1 ${ }^{\text {S96 }}$ to examine whether any had an effect on Yrr1 function, potentially through phosphorylation. T775 and S759 were the only two variable residues

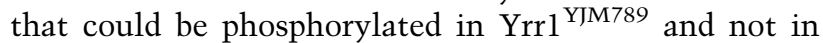
$\mathrm{Yrr}^{\mathrm{S} 96}$. To determine whether T775 has a role in Yrr1 4NQO resistance, we made site-directed mutations in Yrr1 $^{\text {YJM789 }}$ and Yrr1 ${ }^{\text {S96 }}$ (Fig. 4B). Serine and threonine are amino acids with polar uncharged side chains. When threonine and serine are phosphorylated, they become strongly negatively charged. Mutating threonine and serine to glutamate mimics that strong negative charge of phosphorylation. Mutating these residues to an alanine, a nonpolar amino acid, would prevent a negative charge. We tested the effect of site-directed mutation by mutating variable residues on plasmid-encoded Yrr1 to glutamate (E) or alanine (A) and testing the growth of transformed S96 yrr14. Mutation of amino acid T775 to $\mathrm{E}$ in both the $\mathrm{Yrr}^{\mathrm{YJM} 789}$ and $\mathrm{Yrr}^{\mathrm{S} 96}$ alleles increased 4NQO resistance to even greater than the wild-type Yrr1 $^{\text {YJM789 (Fig. 4B). Expression of the yrr1 }}{ }^{\text {YJM789 }}$ T775E allele conferred greater 4NQO resistance than yrr1 ${ }^{\text {YJM789 }}$ T775A or Yrr1 ${ }^{\text {YJM789 }}$. Therefore, yrr1 ${ }^{\text {YJM789 }}$ T775E and yrr1 $^{\text {S96 }}$ T775E were hypomorphic alleles. yrr1 ${ }^{\text {YJM789 }}$ T775A and yrr1 ${ }^{\text {S96 }}$ I775A did not change 4NQO resistance compared with the respective wild-type Yrrl alleles. The yrr1 ${ }^{\text {YJM789 }}$ S759E mutation did not significantly change the $4 \mathrm{NQO}$ resistance of the strain, while the yrr1 ${ }^{\text {YJM789 }}$ S759A resulted in greater 4NQO sensitiv- ity. Mutating 775 to a negatively charged amino acid conferred greater 4NQO resistance than the resistant Yrr1 ${ }^{\text {YJM789 }}$ allele alone. We postulate that while T775 could be the major residue of interaction, the increased sensitivity of yeast expressing yrr1 ${ }^{\text {YJM789 }}$ S759A suggests that both residues are sites of regulation. We made a double mutant (yrr1 ${ }^{\text {YJM789 }}$ S759A T775A), expressed it in S96 yrr1s yeast, and found that the yeast were more sensitive than with $\mathrm{Yrrl}^{\mathrm{YJM} 789}$ bearing either single mutation but not as sensitive as $\mathrm{Yrr}^{\mathrm{S}}{ }^{\mathrm{S} 6}$-expressing yeast (Fig. 4C).

We examined two variable residues of Yrrl that are potential phosphorylation sites and mutated them to glutamate to change the charge. Only one other site in Yrr1 varies in a way that changes the charge of the protein. Amino acid 673 is a glutamate in Yrr1 ${ }^{\text {YMM789 }}$ and a glycine in $\mathrm{Yrr}^{\mathrm{S} 96}$, giving rise to different charges at this site. To investigate the impact of amino acid 673 between the different alleles of Yrr1, we mutated that site and tested the 4NQO resistance. S96 yrr1 10 yeast expressing yrr1 ${ }^{\mathrm{YJM} 789}$ E673G were as 4NQO-sensitive as yeast expressing Yrr1 ${ }^{\text {S96 }}$ (Fig. 4B). However, the converse was not true: Yeast expressing yrr1 ${ }^{\text {S96 }}$ G673E were as 4NQOsensitive as $\mathrm{Yrr}^{\mathrm{S} 96}$-expressing yeast. We further investigated these mutants by introducing mutations at 775 (Fig. 4D). Both the yrr1 ${ }^{\text {YJM789 }}$ T775E and yrr1 ${ }^{\text {S96 }}$ I775E alleles conferred strong 4NQO resistance to yeast. yrr1 ${ }^{\text {YJM789 }}$ E673G converted this allele from YJM789 to a 4QNO-sensitive one, and combining this mutation with T775E restored 4NQO resistance. The double mutants of both alleles (yrr1 ${ }^{\text {YJM789 }}$ E673G T775E and yrr ${ }^{\text {S96 }}$ G673E I775E) were converted to 4NQO-resistant alleles. Consistent with multiple post-translational modifica-
A.

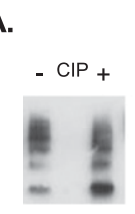

B.

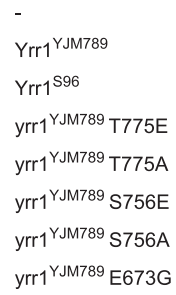

YPD

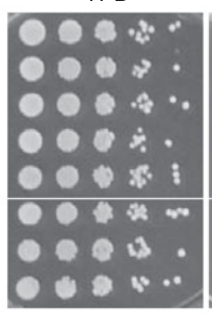

$0.3 \mu \mathrm{g} / \mathrm{ml} 4 \mathrm{NQO}$

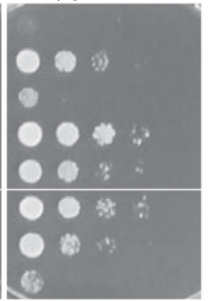

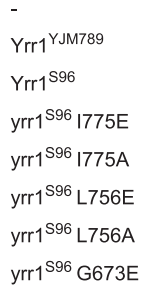

D.
C.

Yrr1 ${ }^{\text {YJM789 }}$

$\mathrm{Yrr1}^{\mathrm{S96}}$

yrr1 ${ }^{\text {YJM789 }}$ S759A T775A

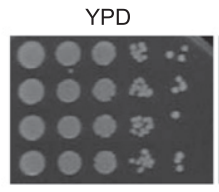

$0.3 \mu \mathrm{g} / \mathrm{ml} 4 \mathrm{NQO}$

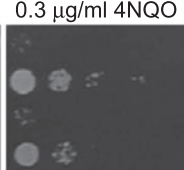

\section{D.}
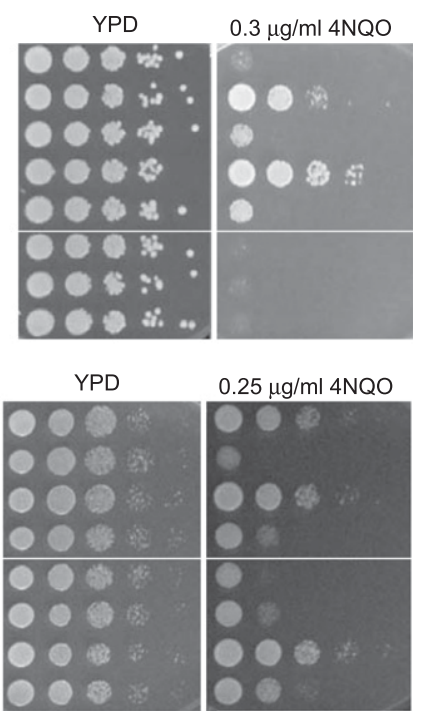

Figure 4. Variation in a single amino acid of Yrr1 ${ }^{\text {YJM789 }}{ }^{\text {contributed to } 4 \text { NQO resistance. }(A) \text { Immunoprecipitation of pMyc-Yrr1 }}{ }^{\text {YJM789 }}$ from S96 lysate treated overnight with CIP. (B) Tenfold serial dilution of S96 yrr1 4 carrying either plasmid with the indicated single amino acid mutant alleles of $\mathrm{Yrrl}^{\mathrm{YJM} 789}$ or $\mathrm{Yrrl}^{\mathrm{S} 96}$ on YPD and YPD $+0.3 \mu \mathrm{g} / \mathrm{mL}$ 4NQO plates for $2 \mathrm{~d}$ at $28^{\circ} \mathrm{C}$ before being photographed. The mutations 775E, 775A, 756E, and 756A were generated in both alleles of Yrr1. The amino acid at position 673 was polymorphic between the two alleles and was switched in Yrr1 alleles to generate yrr1 ${ }^{\text {YJM798 }}$ E673G and yrr1 ${ }^{\text {S96 }}$ G673E alleles. S96 yrr1 $\Delta$ with an empty plasmid is noted with a dash at the top of each plate. (C) A double mutant of yrr1 $1^{\text {YJM } 789}$ S796A and T775A was also transformed into $S 96$ yrr1 $\Delta$ yeast, and the resistance to $0.3 \mu \mathrm{g} / \mathrm{mL} 4 \mathrm{NQO}$ was tested. (D) Double amino acid substitutions at positions 673 and 775 as indicated were generated for each Yrrl allele, and the growth on $0.25 \mu \mathrm{g} / \mathrm{mL}$ 4NQO was tested. 
tions of Yrr1, there were multiple variable amino acids that regulate Yrrl's response to 4NQO.

\section{Assessing the effect of yrr $1^{\text {S96 }} I 775 E$ on the genomic localization}

Mutation of the variable amino acid 775 to a glutamate increased the $4 \mathrm{NQO}$ resistance above the $4 \mathrm{NQO}$ resistance allele $\mathrm{Yrr}^{\mathrm{YJM} 789}$. This mutation is in the C-terminal region of the protein and is not predicted to affect the Zn finger-binding domain (Fig. 3A). To test whether the yrr $1^{\mathrm{S} 96}$ I775E allele would change the genomic location of the TF, we mapped the chromatin-binding sites for the different Yrrl alleles using chromatin immunoprecipitation (ChIP) followed by Illumina sequencing (ChIP-seq), and the reads were mapped back to the reference genome using CisGenome (Supplemental Table 2; Johnson et al. 2007; Robertson et al. 2007; Ji et al. 2008). N-terminal 13xMyc-tagged Yrr1 alleles were expressed from plasmids that were transformed into S96 yrr1s; this design avoided complications due to differences in genetic backgrounds and also ensured that the only Yrr1 allele expressed was the one present on the plasmid. S96 yrr14 strains carrying either $\operatorname{Yrr} 1^{\text {YJM789 }}$, Yrr1 ${ }^{\text {S96 }}$, or yrr1 ${ }^{\text {S96 }}$ I775E were grown in YPD only or in YPD and then shifted to $4 \mathrm{NQO}$ for $2.5 \mathrm{~h}$. Sequenced reads were obtained from three independent biological replicate experiments, along with "input" controls (which gave little background, as described previously; Lefrancois et al. 2009). Across the three strains and two conditions, a total of 329 peaks were identified using CisGenome (Ji et al. 2008) as potential binding sites of Yrr1 (Supplemental Table 3). Cluster analysis was performed on the ChIP-seq peaks to assess any similarities in patterns of TF-binding. The $\operatorname{yrr}^{\mathrm{S} 96}$ I775E peaks from YPD-and-4NQO-treated cells clustered with Yrr1 ${ }^{\text {YJM789 }}$ peaks from 4NQO-treated cells but not with $\mathrm{Yrrl}^{\mathrm{S} 96}$ peaks from YPD- or 4NQO-grown cells (Fig. 5A). While these patterns of Yrr1 binding were the most similar, there were additional loci bound by $\mathrm{Yrr}^{\mathrm{S} 96}$ I775E in 4NQO-treated cells not seen in Yrr1 $^{\text {S96 }}$ I775E from YPD-grown cells. There is a group of loci bound by

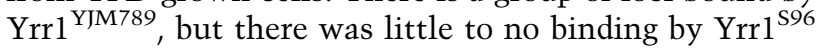
I775E when cells were grown in YPD or 4NQO. This suggests that although amino acid 775 played the major role in regulating 4NQO responses, some other variable amino acids also contributed to the binding variation of Yrr1 in response to 4NQO.

To assess the impact of $\mathrm{Yrr}^{\mathrm{S} 96}$ on individual loci, three loci were closely examined for each condition and allele. We saw that the mutation I775E converted $\mathrm{Yrr}^{\mathrm{S} 96}$ to mimicking the behavior of Yrr1 ${ }^{\text {YJM789 }}$ allele in cells grown

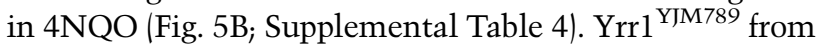
cells grown in 4NQO (but not in YPD alone) as well as

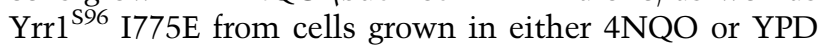
alone bound upstream of YAR068W. The peaks of

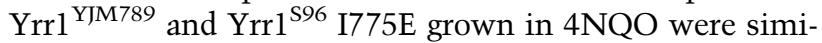
lar, while the peak from $\operatorname{Yrr}^{\mathrm{S} 96}$ I775E grown in YPD was narrower. Expression of YAR068w is induced in petite cells (respiration-deficient yeast) (Epstein et al. 2001). The peak over coding region of FMP52, a gene encoding a mitochondrial protein that is induced in DNA damage (Dardalhon et al. 2007), also shifted between alleles and conditions.

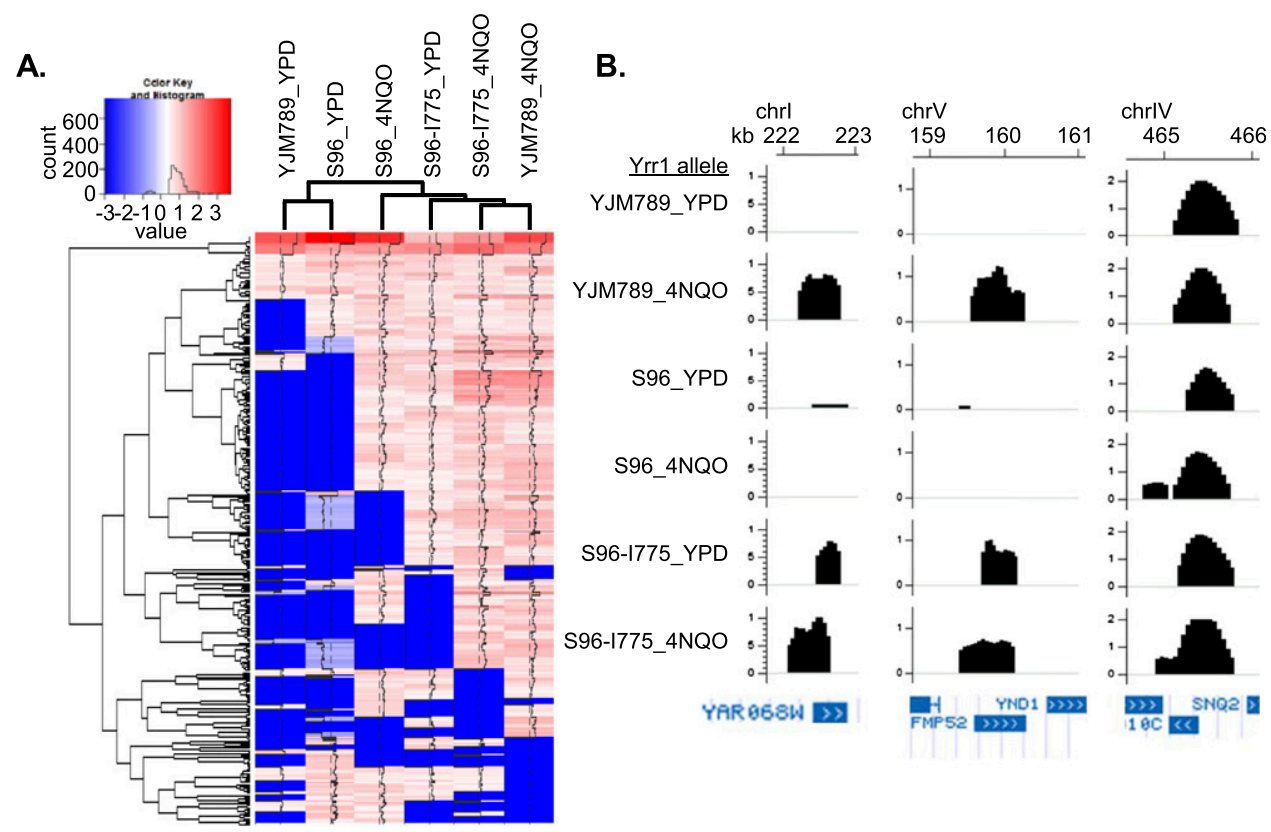

Figure 5. Genomic locations of $y r r 1^{\mathrm{S} 96} \mathrm{I} 775$ compared with $\mathrm{Yrrl}^{\mathrm{S} 96}$ and $\mathrm{Yrr} 1^{\mathrm{YJM} 789}$ grown in 4NQO. Yrr1 targets were mapped in the S96 yrr1s indicated yeast strains using ChIP-seq. (A) Heat map of overlapping peak regions across different alleles and conditions. Log 2 fold changes $\left[\log _{2}(\mathrm{FCs})\right]$ between normalized ChIP and control reads for each overlapping peak region were determined and compared across different yeast strains. A list of the overlapping peak regions and their $\log _{2}(\mathrm{FC})$ values are provided in Supplemental Table 4. $(B)$ Yrr1 ChIP-seq signal tracks from three regions of the genome, including SNQ2 and YAR068W and between FMP52 and PMI40. The $Y$-axis is $\log _{2}(\mathrm{FC})$, and the $X$-axis is the chromosome location with the chromosome and coordinates of each region noted. 
$\mathrm{Yrr1}^{\mathrm{YJM} 789}$ in $4 \mathrm{NQO}, \mathrm{yrr1}^{\mathrm{S} 96}$ I775E in YPD, and yrr1 ${ }^{\mathrm{S} 96}$ I775E in 4NQO did bind known transcriptional targets; for example, the promoter of SNQ2. Snq2 is an ABC plasma membrane transporter that is required for resistance to many chemicals, including 4NQO (Servos et al. 1993) and reactive oxygen species (ROS) (Ververidis et al. 2001), and is known to be regulated by Yrr1 (Le Crom et al. 2002). For many loci, mutation of isoleucine 775 to glutamate in the S96 allele of Yrr1 mimicked 4NQO treatment of Yrr1 ${ }^{\text {YJM789. }}$

\section{Mapping Yrr1-binding regions across genetically diverged yeast strains}

The differences in phenotypes conferred by the YRR1 alleles from the two strain backgrounds could be due to a number of possibilities. To determine how much the binding of Yrr1 could change in both 4NQO and glycerol, we therefore mapped the binding sites of Yrrl in the five different strain backgrounds. Yrr1 from AWRI1631, RM11, and YJM339 contains other sequence differences compared with S96 and YJM789 that alter binding to distinct gene targets. For each strain, targets were mapped in cells grown under three different conditions: rich medium (YPD), 4NQO, and glycerol. The sequencing reads were generated and mapped directly to their respective yeast genomes to avoid mapping biases (see the Materials and Methods; Supplemental Table 5). Significant Yrrl peaks were scored with CisGenome (Ji et al. 2008) using a variety of parameters (Supplemental Table 6). For the results described below, we used a reasonably stringent threshold that provided a low false discovery rate (FDR; $1 \%$ ); qualitatively similar results were observed using a range of different thresholds (see the Supplemental Material for further discussion).

Overall, we identified 125-411 binding regions in the different yeast strains and conditions (Table 1); in general, the highest number of total peaks was identified in cells grown in YPD ( $n=411-256)$, and fewer were found in cells grown in the presence of 4NQO $(n=376-125)$ or glycerol $(n=387-130)$. Analysis of all of the genes associated with Yrrl-bound regions from all ChIP-seq experiments showed enrichment for genes involved in drug response even when cells were grown in the absence of drugs in rich medium or glycerol (Supplemental Tables 7, 8).

\section{Yrr1 has different gene targets in different yeast backgrounds and conditions}

Analysis of Yrrl-binding regions revealed that there are extensive differences in both the different yeasts grown under the same condition and the same yeast grown under different conditions. To some extent, most peak regions bound by Yrrl changed between either strains or conditions. Examples of these variations are shown in Figure 6A.

The peak region in the intergenic space between YNG1 and CYT1, which encode a subunit of the NuA3 histone acetyltransferase and cytochrome $\mathrm{c} 1$, respectively, decreased in AWRI1631, RM11, and YJM339 in 4NQO and glycerol media relative to S96 and YJM789. Cyt1 localizes to the mitochondria and has a role in heme biosynthesis. Varied binding patterns upstream of either gene could be partially responsible for the observed phenotype. An example is the Yrrl peak upstream of SNQ2, which is one of the strongest peaks in most strains and did not vary between different alleles of Yrr1 seen in Figure 5B. The peak was strongest in AWRI1631 grown in YPD and YJM789 grown in glycerol, while peaks in other strains and conditions were about half as strong. The pattern of peaks upstream of GIS1 was similar across all conditions in AWRI1631, RM11, and YJM339 but varied greatly between S96 and YJM789. Gis1 is a Zn finger TF with a jumonji demethylase domain regulating phospho-

Table 1. Yrr1 ChIP target and overlaps between strains

\begin{tabular}{|c|c|c|c|c|c|c|c|}
\hline Medium & Strain & Peaks & YJM789 & S96 & AWRI1631 & RM11 & YJM339 \\
\hline YPD & YJM789 & 277 & 1 & 6 & 14 & 5 & 9 \\
\hline YPD & S96 & 411 & 21 & 2 & 39 & 7 & 9 \\
\hline YPD & AWRI1631 & 262 & 11 & 17 & 3 & 11 & 11 \\
\hline YPD & RM11 & 387 & 31 & 9 & 28 & 3 & 31 \\
\hline YPD & YJM339 & 259 & 7 & 17 & 3 & 11 & 12 \\
\hline 4NQO & YJM789 & 148 & 0 & 33 & 18 & 9 & 7 \\
\hline 4NQO & S96 & 125 & 34 & 10 & 51 & 44 & 23 \\
\hline 4NQO & AWRI1631 & 138 & 42 & 33 & 0 & 0 & 4 \\
\hline 4NQO & RM11 & 131 & 8 & 40 & 5 & 11 & 1 \\
\hline 4NQO & YJM339 & 376 & 49 & 64 & 0 & 0 & 4 \\
\hline Glycerol & YJM789 & 130 & 59 & 60 & 89 & 61 & 61 \\
\hline Glycerol & S96 & 144 & 39 & 2 & 79 & 18 & 14 \\
\hline Glycerol & AWRI1631 & 138 & 42 & 0 & 0 & 0 & 0 \\
\hline Glycerol & RM11 & 131 & 47 & 10 & 29 & 0 & 2 \\
\hline Glycerol & YJM339 & 387 & 49 & 64 & 0 & 0 & 4 \\
\hline
\end{tabular}

Number and percentage of unique peaks for each pairwise strain/condition combination. Off-diagonal entries are the number of peaks substantially different $\left(\log _{2}\right.$ normalized signal threshold) in the two-way comparison; e.g. in the cell at the intersection of row S96 (411) and column YJM339, 9\% of the 411 binding regions in S96 are different in the two-way comparison of S96 and YJM339 under YPD growth condition. Along diagonal entries are the number of unique binding regions (shaded) in one strain but different from the other four strains under each condition; e.g. at the intersection of row S96 (411) and column S96, 2\% of the 411 binding regions in S96 are different from other binding regions in the other four strains under YPD growth condition. 
Gallagher et al.

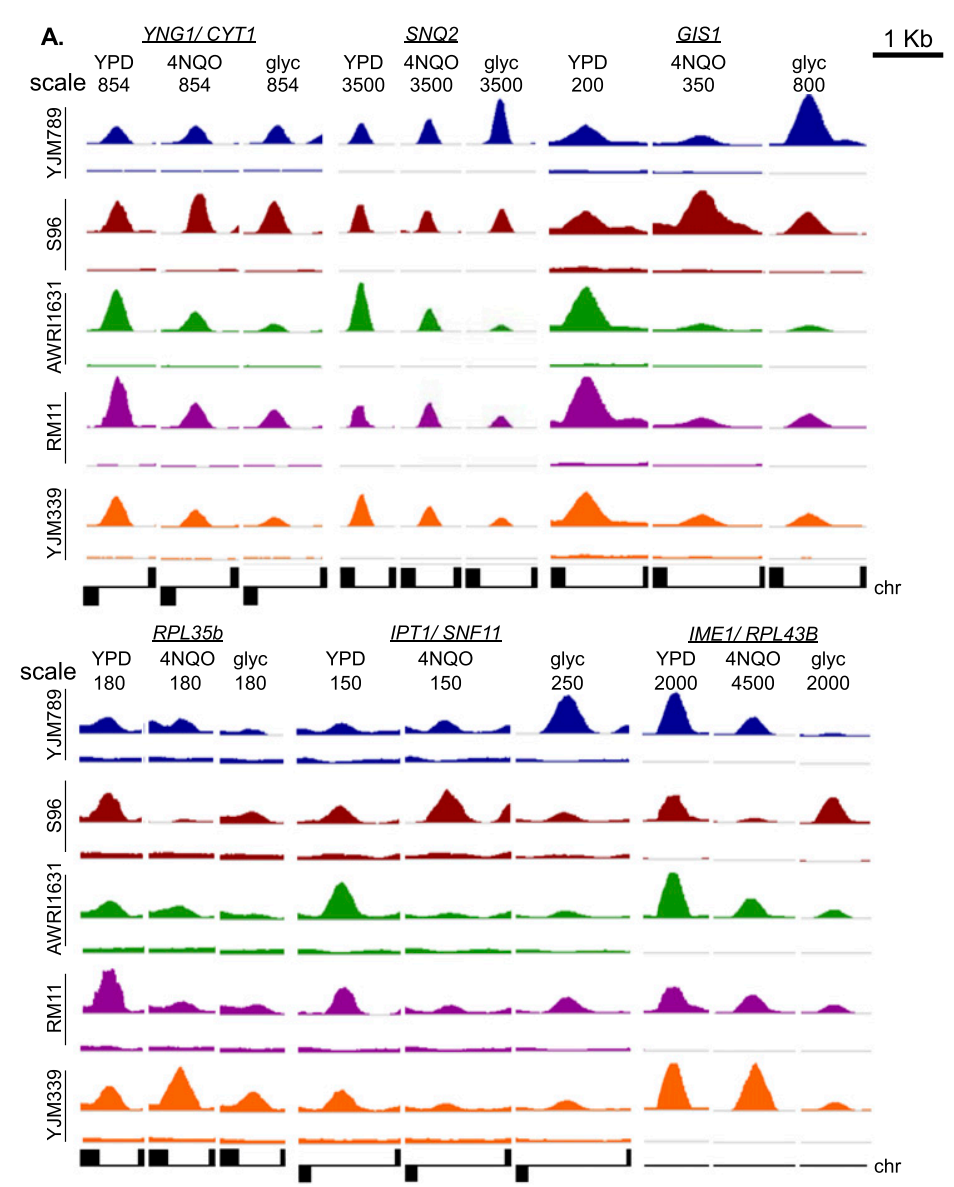

B. RPC82/QCR2 IBS1/APD1 GIP3/ISU1 ASK1/SFK1 SNG1/YPP1 ELR1 YPR152C/PDR5 $1 \mathrm{~Kb}$

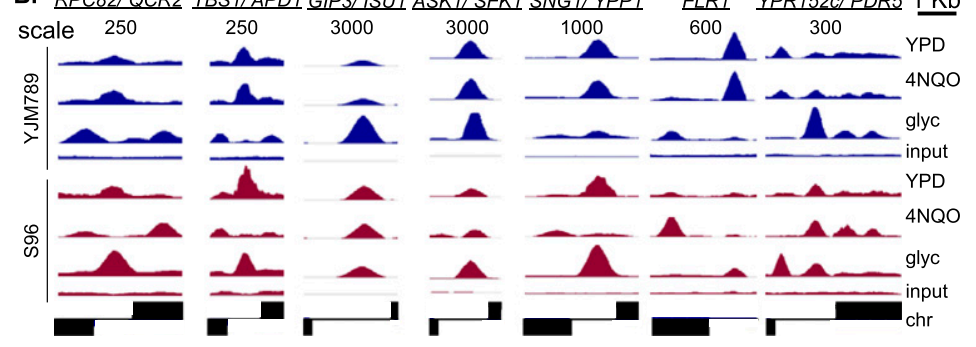

Figure 6. Differential binding at distinct targets from five different strains in three different growth conditions. (A) Yrrl ChIP-seq signal tracks from YJM789 (blue), S96 (red), AWRI1631 (green), RM11 (purple), and YJM339 (orange) were drawn for peak regions in YPD, $4 \mathrm{NQO}$, and glycerol media with the input tracks below each ChIP track. Genes with $5^{\prime}$ ends downstream from peak regions ae indicated above the signal tracks, and corresponding regions on chromosomes are shown below. The black line represented the chromosome, and the scale is noted by a bar demonstrating $1 \mathrm{~kb}(X$ axis). ORFs are boxed in black. The scale for each signal track for a condition was drawn to fit and is noted above the signal tracks (Y-axis). (B) Yrrl ChIP-seq signal tracks from YJM789 (blue) and S96 (red) from the three different conditions were drawn in the same scale for each peak region ( $Y$-axis). The same schematic was used as described above to represent genes. Note that differential Yrrl binding was observed for the different yeasts; in some cases, binding is similar under one condition but different in another (e.g., QCR2 in YPD compared with glycerol); in other cases, binding is different in all conditions (e.g., FLR1). lipids levels (Oshiro et al. 2003), transcription of starvation genes, and DNA damage response (Jang et al. 1999). The GIS1 peak was similar across different strains in YPD but increased in YJM789 grown in glycerol and also in S96 grown in 4NQO compared with other strains grown in the same conditions. The binding pattern between IPT1 and SNF11 was similar to that observed for GIS1. Snf11 is a component of the Swi/Snf chromatin remodeling complex (Treich et al. 1995) and Ipt1, an inositolphosphotransferase that has a role in drug resistance (Hallstrom and Moye-Rowley 2000). The binding pattern of the Yrr1 upstream two genes encoding ribosomal proteins also was similar to the strongest binding observed in YJM339 grown in 4NQO. Overall, the most variation in the number of peaks among the different yeast was observed in 4NQO $(\mathrm{STD}=107.9)$ and glycerol $(\mathrm{STD}=112.5)$ as compared with $\mathrm{YPD}(\mathrm{STD}=73.6)$.
It is possible that the variation in Yrrl binding across all strains was due to an overall reduced level of Yrrl's ability to bind DNA in different strains. However, we do not believe that this is the case because we observed some peaks with similar levels among the different strains (e.g., the intergenic peak between YNG1 and CYT1 varied less than twofold for strains grown in YPD) and because we observed some very strong peaks in any given strain and condition (e.g., between IME1 and RPL43b). We also performed a systematic analysis, as described in the next section.

\section{Varied patterns of binding across different strains} and conditions revealed by systematic analysis

To compare all peaks among the different strains, we used the stringent criteria described in the Materials and 
Methods and a pipeline similar to that described previously (Kasowski et al. 2010; Zheng et al. 2010). We first identified the regions that bound Yrrl in the different strains and determined the normalized signals in these regions for each of these strains. We then analyzed the differences in signals as well as the percentage overlap of the binding regions. We first determined the correlation of signals in all Yrr1-bound regions between (1) the same strain grown in different conditions (Supplemental Fig. 8), (2) different strains in the same condition, and (3) as a control, the independent biological replicates from the same strain. The individual comparisons are presented in Supplemental Figures S4 and S5, and the results are summarized in Figure 7A. For the Yrr1-bound regions, the correlations in normalized signals between different strains and different conditions were significantly lower than the correlations between biological replicates (Wilcoxon rank-sum test, $P=0.02635$ and $P=0.02042$, respectively) (Fig. 6A), indicating significant variation in binding among the different strains grown in the same condition and the same strain grown under different conditions. Overall, there was just as much variation in Yrrl targets between strains as there was between different conditions.

For each binding target location, we also examined the differences in normalized binding signals for all five strains and all three conditions using the clustering diagram depicted in Figure 6B. As is evident in the figure, extensive Yrr1 target and signal differences exist between each of the different strains irrespective of the growth conditions. However, some strains do appear more similar to one another under the same conditions (e.g., AWRI1631 and RM11 in 4NQO and RM11 and S96 in glycerol) (Supplemental Fig. S5). Interestingly, in at least two cases, the patterns appeared similar for different yeast exposed to different conditions (e.g., YJM789 and S96 grown in glycerol and 4NQO, respectively). These results are also evident from the pairwise correlation analyses (Supplemental Fig. S6) and suggest that the yeast TFs can switch their binding programs.

We also determined the number of binding sites that were unique or substantially different in signal (more than twofold apart in normalized signal) between the different yeast strains grown under both the same and different conditions. Consistent with the heat map results (Table 1), we observed extensive divergence in similar binding sites among the different yeast grown in the same condition as well as for the same yeast grown in different conditions. For example, YJM789 and S96 had $33 \%$ and $40 \%$ unique/different binding sites /more than twofold) for cells grown on glycerol or in the presence of 4NQO, respectively. To ensure that these differences were not due to threshold effects, two analyses were performed. First, we changed the threshold of fold differences and found similar results (data not shown). Second, we examined the signals of peaks that were not found to be overlapping; potentially, many of these might lie just below the threshold. As shown in Supplemental Figure S7, although some peaks may be similar and fall below the threshold in one of the strains, a large number of peaks were very different, and the Yrrl ChIP signal was

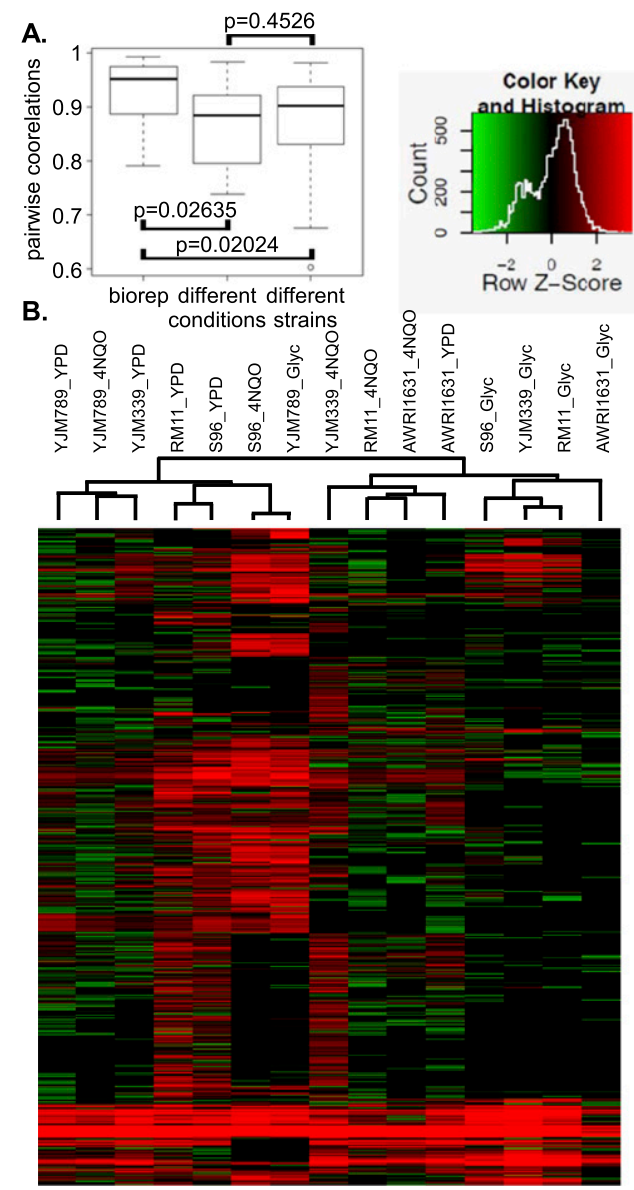

Figure 7. Different binding targets of Yrrl among five yeast strains. Yrrl targets were mapped in five indicated yeast strains using ChIP-seq. (A) A summary of the pairwise correlations between peak signals (log read counts) in ChIP samples of two biological replicates of the same strain (left), the same strain under different conditions (middle), and different strains under the same condition (right). Correlations between different strains grown under the same conditions or the same strains grown under different conditions were significantly different from that observed for biological replicates (significance differences between replicates and conditions [Wilcoxon rank-sum test, $P=0.026$ ] or different strains [Wilcoxon rank-sum test, $P=$ $0.020]$ ), whereas correlations between conditions and between strains were not significantly different (Wilcoxon rank-sum test, $P=0.4526)$. (B) Heat map of overlapping peak regions across different strains and conditions. $\log _{2}$ fold changes between normalized ChIP and control reads for each overlapping peak region were determined and compared across different yeast. A list of the overlapping peak regions and their $\log 2(\mathrm{FC})$ values is provided in Supplemental Table 7.

near background levels in one strain or the other. Overall, these results indicate that both the number of Yrr1 targets and their binding signals often vary among different yeasts.

Extensive variations in Yrrl localization were evident at different loci in the different yeast and conditions. Common overall patterns were evident based on the dendrogram above the heat map (Fig. 7B). In general, Yrrl from cells grown in glycerol had more similar 
patterns of binding than that from cells grown in YPD or 4NQO. The two Yrrl-binding patterns that were the most similar were in S96 grown in 4NQO and in YJM789 grown in glycerol. PDR3, SNQ2, PDR15, YOR1, PDR16, and PDR5, which were strongly associated with Yrr1 in all strains and conditions, were involved in drug response including Yrrl itself (Supplemental Table 8).

From the peaks identified from the ChIP-seq analysis, we also performed a de novo motif search for Yrr1binding sites (see the Materials and Methods) in all strains and growth conditions using MEME and recovered the consensus binding sites (Supplemental Fig. S8). These searches revealed distinct but related consensus binding sites among one another, which were also related to the published consensus (RCGGRDWTWD) (Zhu et al. 2009). In particular, the consensus binding site from S96 in 4NQO was very similar to the binding sites seen in S96, RM11, and YJM339 grown in glycerol. AWRI1631 binding sites from glycerol showed some similarity to YJM789 grown in YPD. In many cases, when there was a similar deduced motif, there was also a similar Yrr1binding pattern (e.g., AWRI1631 in 4NQO and glycerol media). However, overall, we did observe condition- and strain-specific variations, suggesting that the binding preference may differ across different growth conditions.

\section{Discussion}

We identified and characterized a single protein (Yrr1) that affected growth in three different conditions and is polymorphic across diverse yeast strains. The polymorphisms in YRR1 were outside the DNA-binding region, with the nonconservative substitutions located at the $\mathrm{C}$ terminus. One of the residues, at position 775 , was likely the major reason for these different phenotypes, as changing it to a glutamate causes 4NQO resistance in an otherwise sensitive strain. Since conversion to an alanine does not eliminate 4NQO resistance in YJM789, we propose that other residues are responsible for the phenotypic variations as well. Without glutamate at 673 , the Yrr1 ${ }^{\text {YJM789 }}$ could not confer 4NQO resistance without mutation of 775 to a glutamate. Unlike the I775E substitution in $\mathrm{Yrr}^{\mathrm{S} 96}$, mutation of 673 to a glutamate could not convert the $\mathrm{Yrr}^{\mathrm{S} 96}$ allele to a 4NQO-resistant allele. We further propose that these Yrrl amino acid differences lead to variations in association with protein partners/ cofactors such as kinases, which in turn results in selection of different binding targets. Since previous studies have shown in yeast and humans cells that TFs often have different partners at distinct loci that are important for binding (Kasowski et al. 2010; Zheng et al. 2010; Karczewski et al. 2011), it is likely that Yrrl has distinct partners at different targets, varying in the different strains and under the different conditions. A likely candidate is a serine/threonine kinase that phosphorylates polymorphic sites in Yrrl in response to 4NQO. The interaction of regulatory enzymes and their substrates is difficult to characterize and often requires overexpression or in vitro purification to monitor physical interactions (Ruiz et al. 2011). It is interesting that Yrr1 targets are sometimes more similar in different strains grown under different conditions then in either the same strain grown under different conditions or different strains grown in the same conditions. The simplest explanation for this observation is that different factors work with Yrr1 under different strains and conditions and that some of these combinations are shared in those cases. It is likely the amino acid variations that are responsible for these interaction differences, which is consistent with our demonstrating that coding sequence changes are primarily responsible for the phenotypic differences that we observed.

Yrrl is polymorphic among different yeast strains, and we propose that this TF belongs to a class of regulatory proteins that we call "master variators." We suggest that master variators are proteins that have three characteristics: (1) They are polymorphic; (2) they are regulatory proteins such as TFs, proteins kinases, and other modification enzymes; and (3) they affect significant numbers of downstream targets. In contrast to deletions, study of natural variation would likely limit suppressor mutations that would compensate for severe phenotypes (Teng et al. 2013). Variation in Yrr1 and other master regulators can cause significant differences in phenotypes through alteration in regulatory programs rather than simply loss or reduction of function, as has been described previously (Chan et al. 2010). These alterations can improve one phenotype at a cost to another; indeed, the Yrr1 ${ }^{\mathrm{YJM} 789}$ allele improves growth in the presence of $4 \mathrm{NQO}$ but reduces growth in glycerol even when the allele responsible for improved growth on glycerol is present. Variation of Yrr1 between EC1118 and S288c affects the regulation of $S N G 1$ during the fermentation process (Brion et al. 2013). We note that this concept is different from that reported by others in which loss of function causes a distinct phenotype. For Yrr1, we observed a phenotype transformation due to a single gene through molecular reprogramming.

The mechanism by which Yrrl causes distinct phenotypes is not known in detail but may involve differences in many different binding targets. Yrrl binds upstream of many genes affecting mitochondrial function and drug response, and these targets differ in the different strain backgrounds (Supplemental Table 9). For example, Yrr1 is differentially bound upstream of ISU1, QCR2, and TBS1 (genes involved in mitochondrial function in S96 and YJM789) in some conditions, and in other conditions, it binds upstream of PDR5, SNG1, and FLR1 (genes involved in drug response) (Fig. 6B). Yrrl activity correlates with SNG1 and FLR1 expression (Le Crom et al. 2002; Teixeira et al. 2008; Brion et al. 2013; Kodo et al. 2013), and thus Yrr1 is likely a direct activator of gene expression of these targets. Yrr1 binding in YJM789 at these and other regions may facilitate 4NQO resistance. Interestingly, Yrr1 is thought to be a repressor as well as an activator because its activity can affect genes in different ways (Cui et al. 1998; Zhang et al. 2001; Le Crom et al. 2002; Lucau-Danila et al. 2003). Thus, its variation in 
binding at different gene targets can alter expression in different ways; as such, its control over variation as a master variator likely affects gene expression in very different ways and thus leads to the great extent of phenotypic diversity.

In conclusion, we demonstrate that variation in an important TF is responsible for major phenotypic changes in yeast. Previous studies have highlighted the elevated levels of nonsynonymous changes in TFs among different yeast strains (Gu et al. 2005). Since many human TFs and other regulatory proteins are also polymorphic, it is likely that some of these potential master variators may also be responsible for phenotypic differences, and thus this may be a general mechanism for mediating extensive regulatory (e.g., expression and binding QTLs) and phenotypic variation in all eukaryotes.

\section{Materials and methods}

\section{Yeast strains and plasmids}

All yeast strains used in this study are haploids and are described in Supplemental Table 10. S96 (isogenic to S288c) is a laboratory strain, YJM789 was derived from a clinical isolate from the lungs of an AIDS patient (McCusker et al. 1994; Wei et al. 2007), AWRI1631 was derived from a wine strain (Borneman et al. 2008), and RM11-1b was derived from the vineyard diploid by removing $\mathrm{HO}$ and was sporulated, and the $\mathrm{KAN}^{\mathrm{R}}$ was removed as described (Gueldener et al. 2002). YJM339-2d was derived from a clinical isolate from a human bile tube (McCusker et al. 1994), and a stable haploid was generated as RM11-1b. pGS35 $\left(\mathrm{KAN}^{\mathrm{R}}\right)$ and pGS36 $\left(\mathrm{HYG}^{\mathrm{R}}\right)$ are centromeric plasmids that were a gift from Gavin Sherlock. All oligonucleotides used are described in Supplemental Table 11. YRR1 with the endogenous promoter and terminator were cloned by homologous recombination into the $\mathrm{XbaI}$ and SpeI sites into pGS35. The entire transcriptional unit of YRR1 was selected, and the promoter contained four polymorphs, while downstream polymorphisms occured downstream from the transcriptional stop of the YRR1 mRNA (Xu et al. 2009). Yeast were grown under standard conditions and transformed as outlined in Gietz and Schiestl (2007). YRR1 was tagged both at the $\mathrm{C}$ terminus with the $13 \mathrm{xMyc}$ tag marked with KAN $^{\mathrm{R}}$ (Longtine et al. 1998) and at the $\mathrm{N}$ terminus by PCRstitching the $13 \mathrm{xMyc}$ tag to the coding region of YRR1 $1^{Y I M 789}$ and $Y R R 1^{S 96}$ and cloning into pSG35. Endogenous $Y R R 1$ was deleted via homologous recombination with $\mathrm{HYG}^{\mathrm{R}}$ (Goldstein and McCusker 1999), and plasmids were transformed into strains using standard lithium acetate transformation. Plasmids were maintained by addition of $200 \mu \mathrm{g} / \mathrm{mL}$ G418 for selection. Sitedirected mutagenic PCR was carried out with overlapping primers containing each of the mutations and flanking primers used for cloning. The $5^{\prime}$ and $3^{\prime}$ PCR products were then used as templates to PCR-stitch yrr1 with the mutation. yrr1 alleles were cloned into pGS35, and mutations were verified by sequencing the plasmid. Growth rates were calculated by measuring the $\mathrm{OD}_{600}$ of a yeast culture grown to log phase in YPD, washed, and suspended in $30 \mathrm{~mL}$ of $4 \mathrm{NQO}$ or YPglycerol. Readings while the culture was in exponential growth were fitted to a log scale. The doubling time in hours was derived by the equation fitted to the growth curve.

\section{Immunoprecipitation}

Cells were grown in YPD to mid-log phase and then shifted to other medium. Next, $0.25 \mu \mathrm{g} / \mathrm{mL}$ 4NQO dissolved in DMSO (Sigma-Aldrich) was added for $2.5 \mathrm{~h}$ to YPD, and/or cells were washed with YP and shifted to YP + glycerol for $8.5 \mathrm{~h}$. Cells were lysed in the Fastprep for $20 \mathrm{sec}$ at speed 5 and then ice for $5 \mathrm{~min}$ three times in $50 \mathrm{mM}$ Tris $\mathrm{HCl}(\mathrm{pH} 7.8), 150 \mathrm{mM} \mathrm{NaCl}, 4 \%$ glycerol, $1.5 \mathrm{mM} \mathrm{Mg}$ acetate, and $0.15 \%$ NP-40. Clarified extract $(1.5 \mathrm{mg})$ was incubated with $35 \mu \mathrm{L}$ of $50 \%$ anti-Myc EZ-view affinity gel (Sigma-Aldrich) for $2 \mathrm{~h}$ to overnight at $4^{\circ} \mathrm{C}$. Washed immunoprecipitates were boiled with Lammeli sample buffer, separated on $4 \%-12 \%$ polyacrylamide gel, and transferred to nitrocellulose. Westerns were blotted with mouse anti-Myc 9E10 (Sigma). To determine whether there was any background in the immunoprecipitations, a mock immunoprecipitation was carried out with Yrr1-Myc-tagged lysate and protein A-agarose beads. Lysate was treated with $15 \mu \mathrm{L}$ of CIP overnight at $4^{\circ} \mathrm{C}$, and then Yrr1-Myc was immunoprecipitated.

\section{NQO response QTL mapping}

A total of 124 S96/YJM789 segregants (Mancera et al. 2008) was manually scored for growth on 4NQO medium on a scale of $0-5$. Their specific genotypes at 55958 SNP marker loci were filtered (must have more than four strains of either parental genotype) and reduced to 5523 nonredundant genotype blocks. Onedimensional QTL scanning was performed using the R package qtl with EM method. The 95\% Bayes credible interval was calculated for the highest peak on chromosome 15 . The significance threshold was above LOD 3.84 after 1000 permutations, and the peak above YRR1 was the only QTL that passed the threshold. The peak was represented by $10^{\wedge} \mathrm{LOD}$ rescaled to make the area under the curve equal 1.

\section{ChIP-seq}

Cells were grown in YPD to mid-log phase, diluted to early log phase, and split into three cultures. YPD cultures were grown for 2.5 h. 4NQO $(0.25 \mu \mathrm{g} / \mathrm{mL}$ ) dissolved in DMSO (Sigma) was added for $2.5 \mathrm{~h}$. The third culture was washed with YP and shifted to YP + glycerol for $8.5 \mathrm{~h}$. Thirty-five OD units were cross-linked with $1 \%$ formaldehyde for $45 \mathrm{~min}$ before being washed once with water and flash-frozen. ChIP was carried out as described (Lefrancois et al. 2009) with the following modifications: The addition of glycine was omitted, and $35 \mu \mathrm{L}$ of $50 \%$ anti-Myc EZview affinity gel was added (Sigma-Aldrich). Two-hundred nanograms of chromatin was used as input controls. The construction of multiplexed barcoded libraries permitted the simultaneous sequencing of 24 samples in the Illumina HiSeq sequencers (Lefrancois et al. 2009). Libraries were sequenced on Illumina HiSeq as 100-base-pair (bp) paired-end reads. For different allelic ChIP of pMyc-Yrrl, there were three biological replicates for ChIP of each treatment and one replicate for input. The average generated reads were $3,357,478$, and the alignment rate was $99.17 \%$ for ChIP samples; the average generated reads were $3,134,818$, and the alignment rate was $98.70 \%$ for input. ChIP from different strains with Yrr1-Myc tag was carried out as described (Aparicio et al. 1991). Two to three biological replicates of genetically divergent strain ChIP samples were sequenced on Illumina GAII as 32-bp single-end reads, generating an average of 2.66 million post-filtered reads, with $64 \%$ mapping back to the genome; the input samples generated an average of 3.55 million post-filtered reads, with $77 \%$ mapping back to the genome. Normalized peak signals on $\log _{2}$ scale for all strains and conditions were clustered by Euclidian distance using heatmap. 2 function in R.

\section{Acknowledgments}

Sequencing libraries were prepared by Lixia Jiang. Sequencing was carried out in the Stanford Genome Center. Amna Khan, Lihua Jiang, Linfeng Wu, and Randall Mann provided technical 
assistance and advice. E. Miriami and B.D. gave welcomed comments on this manuscript. This research was funded by National Institutes of Health (NIH) grant CA077808. J.E.G.G. was funded by NIH Stanford Genetic Training grant 5T32HG000044. N.M. was supported by the Stanford Summer Research Program in Biomedical Sciences/Amgen Scholars Program. J.E.G.G. designed and directed the study with the aid of M.P.S. J.E.G.G., B.D., and M.P.S. wrote the paper. J.E.G.G. constructed all strains and plasmids for this study. J.E.G.G. did the chemical screen and scored 4NQO responses (Fig. 1A,B; Supplemental Figs. 1, 2), and W.Z. determined the responsible genetic loci with linkage analysis (Fig. 1C; Supplemental Table 1). J.E.G.G. tested the growth of all strains with YRR1 alleles, expression levels of Yrrl in different conditions, and mutant analysis (Figs. 2-4). J.E.G.G. and N.M. carried out ChIP-seq on the five different strains. J.E.G.G. and B.D. contributed to Figure 5. X.R. called and correlated peaks in Figure 5 and Supplemental Tables 3 and 4. W.Z. and H.Z. called and correlated peaks and deduced the Yrrl motifs under the direction of H.Z. (Figs. 6, 7; Supplemental Figs. 3-9; Supplemental Table 5-8).

\section{References}

Aparicio OM, Billington BL, Gottschling DE. 1991. Modifiers of position effect are shared between telomeric and silent mating-type loci in S. cerevisiae. Cell 66: 1279-1287.

Borneman AR, Leigh-Bell JA, Yu H, Bertone P, Gerstein M, Snyder M. 2006. Target hub proteins serve as master regulators of development in yeast. Genes Dev 20: 435-448.

Borneman AR, Forgan AH, Pretorius IS, Chambers PJ. 2008. Comparative genome analysis of a Saccharomyces cerevisiae wine strain. FEMS Yeast Res 8: 1185-1195.

Brem RB, Yvert G, Clinton R, Kruglyak L. 2002. Genetic dissection of transcriptional regulation in budding yeast. Science 296: 752-755.

Brion C, Ambroset C, Sanchez I, Legras JL, Blondin B. 2013. Differential adaptation to multi-stressed conditions of wine fermentation revealed by variations in yeast regulatory networks. BMC Genomics 14: 681.

Chan YF, Marks ME, Jones FC, Villarreal G Jr, Shapiro MD, Brady SD, Southwick AM, Absher DM, Grimwood J, Schmutz J, et al. 2010. Adaptive evolution of pelvic reduction in sticklebacks by recurrent deletion of a Pitx1 enhancer. Science 327: 302-305.

Cui Z, Shiraki T, Hirata D, Miyakawa T. 1998. Yeast gene YRR1, which is required for resistance to 4-nitroquinoline $\mathrm{N}$-oxide, mediates transcriptional activation of the multidrug resistance transporter gene SNQ2. Mol Microbiol 29: 1307-1315.

Dardalhon M, Lin W, Nicolas A, Averbeck D. 2007. Specific transcriptional responses induced by 8-methoxypsoralen and UVA in yeast. FEMS Yeast Res 7: 866-878.

Davidson EH. 2011. Evolutionary bioscience as regulatory systems biology. Dev Biol 357: 35-40.

Ehrenreich IM, Torabi N, Jia Y, Kent J, Martis S, Shapiro JA, Gresham D, Caudy AA, Kruglyak L. 2010. Dissection of genetically complex traits with extremely large pools of yeast segregants. Nature 464: 1039-1042.

Epstein CB, Waddle JA, Hale Wt, Dave V, Thornton J, Macatee TL, Garner HR, Butow RA. 2001. Genome-wide responses to mitochondrial dysfunction. Mol Biol Cell 12: 297-308.

Gerke J, Lorenz K, Cohen B. 2009. Genetic interactions between transcription factors cause natural variation in yeast. Science 323: 498-501.

Gerke J, Lorenz K, Ramnarine S, Cohen B. 2010. Gene-environment interactions at nucleotide resolution. PLoS Genet 6: e1001144.
Gietz RD, Schiestl RH. 2007. Frozen competent yeast cells that can be transformed with high efficiency using the LiAc/SS carrier DNA/PEG method. Nat Protoc 2: 1-4.

Goldstein AL, McCusker JH. 1999. Three new dominant drug resistance cassettes for gene disruption in Saccharomyces cerevisiae. Yeast 15: 1541-1553.

Gu Z, David L, Petrov D, Jones T, Davis RW, Steinmetz LM. 2005. Elevated evolutionary rates in the laboratory strain of Saccharomyces cerevisiae. Proc Natl Acad Sci 102: 1092-1097.

Guan W, Jiang H, Guo X, Mancera E, Xu L, Li Y, Steinmetz L, Li $\mathrm{Y}, \mathrm{Gu}$ Z. 2010. Antagonistic changes in sensitivity to antifungal drugs by mutations of an important ABC transporter gene in a fungal pathogen. PLOS ONE 5: e11309.

Gueldener U, Heinisch J, Koehler GJ, Voss D, Hegemann JH. 2002. A second set of loxP marker cassettes for Cre-mediated multiple gene knockouts in budding yeast. Nucleic Acids Res 30: e23.

Hallstrom TC, Moye-Rowley WS. 2000. Multiple signals from dysfunctional mitochondria activate the pleiotropic drug resistance pathway in Saccharomyces cerevisiae. J Biol Chem 275: 37347-37356.

Infante CR, Park S, Mihala AG, Kingsley DM, Menke DB. 2013. Pitx1 broadly associates with limb enhancers and is enriched on hindlimb cis-regulatory elements. Dev Biol 374: 234-244.

Jang YK, Wang L, Sancar GB. 1999. RPH1 and GIS1 are damageresponsive repressors of PHR1. Mol Cell Biol 19: 7630-7638.

Ji H, Jiang H, Ma W, Johnson DS, Myers RM, Wong WH. 2008. An integrated software system for analyzing ChIP-chip and ChIP-seq data. Nat Biotechnol 26: 1293-1300.

Johnson DS, Mortazavi A, Myers RM, Wold B. 2007. Genomewide mapping of in vivo protein-DNA interactions. Science 316: $1497-1502$.

Karczewski KJ, Tatonetti NP, Landt SG, Yang X, Slifer T, Altman RB, Snyder M. 2011. Cooperative transcription factor associations discovered using regulatory variation. Proc Natl Acad Sci 108: 13353-13358.

Kasowski M, Grubert F, Heffelfinger C, Hariharan M, Asabere A, Waszak SM, Habegger L, Rozowsky J, Shi M, Urban AE, et al. 2010. Variation in transcription factor binding among humans. Science 328: 232-235.

Kodo N, Matsuda T, Doi S, Munakata H. 2013. Salicylic acid resistance is conferred by a novel YRR1 mutation in Saccharomyces cerevisiae. Biochem Biophys Res Commun 434: 42-47.

Le Crom S, Devaux F, Marc P, Zhang X, Moye-Rowley WS, Jacq C. 2002. New insights into the pleiotropic drug resistance network from genome-wide characterization of the YRR1 transcription factor regulation system. Mol Cell Biol 22: 2642-2649.

Lefrancois P, Euskirchen GM, Auerbach RK, Rozowsky J, Gibson T, Yellman CM, Gerstein M, Snyder M. 2009. Efficient yeast ChIP-seq using multiplex short-read DNA sequencing. BMC Genomics 10: 37.

Liti G, Carter DM, Moses AM, Warringer J, Parts L, James SA, Davey RP, Roberts IN, Burt A, Koufopanou V, et al. 2009. Population genomics of domestic and wild yeasts. Nature 458: $337-341$.

Longtine MS, McKenzie A 3rd, Demarini DI, Shah NG, Wach A, Brachat A, Philippsen P, Pringle JR. 1998. Additional modules for versatile and economical PCR-based gene deletion and modification in Saccharomyces cerevisiae. Yeast 14: 953-961.

Lucau-Danila A, Delaveau T, Lelandais G, Devaux F, Jacq C. 2003. Competitive promoter occupancy by two yeast paralogous transcription factors controlling the multidrug resistance phenomenon. J Biol Chem 278: 52641-52650. 
Mancera E, Bourgon R, Brozzi A, Huber W, Steinmetz LM. 2008. High-resolution mapping of meiotic crossovers and noncrossovers in yeast. Nature 454: 479-485.

McCusker JH, Clemons KV, Stevens DA, Davis RW. 1994. Genetic characterization of pathogenic Saccharomyces cerevisiae isolates. Genetics 136: 1261-1269.

Mortimer RK, Romano P, Suzzi G, Polsinelli M. 1994. Genome renewal: A new phenomenon revealed from a genetic study of 43 strains of Saccharomyces cerevisiae derived from natural fermentation of grape musts. Yeast 10: 1543-1552.

Oshiro J, Han GS, Iwanyshyn WM, Conover K, Carman GM. 2003. Regulation of the yeast DPP1-encoded diacylglycerol pyrophosphate phosphatase by transcription factor Gislp. I Biol Chem 278: 31495-31503.

Rebeiz M, Pool JE, Kassner VA, Aquadro CF, Carroll SB. 2009. Stepwise modification of a modular enhancer underlies adaptation in a Drosophila population. Science 326: 1663-1667.

Robertson G, Hirst M, Bainbridge M, Bilenky M, Zhao Y, Zeng T, Euskirchen G, Bernier B, Varhol R, Delaney A, et al. 2007. Genome-wide profiles of STAT1 DNA association using chromatin immunoprecipitation and massively parallel sequencing. Nat Methods 4: 651-657.

Ruiz A, Xu X, Carlson M. 2011. Roles of two protein phosphatases, Reg1-Glc7 and Sit4, and glycogen synthesis in regulation of SNF1 protein kinase. Proc Natl Acad Sci 108: 6349-6354.

Servos J, Haase E, Brende, M. 1993. Gene SNQ2 of Saccharomyces cerevisiae, which confers resistance to 4-nitroquinoline$\mathrm{N}$-oxide and other chemicals, encodes a $169 \mathrm{kDa}$ protein homologous to ATP-dependent permeases. Mol Gen Genet 236: $214-218$.

Teixeira MC, Dias PJ, Simoes T, Sa-Correia I. 2008. Yeast adaptation to mancozeb involves the up-regulation of FLR1 under the coordinate control of Yap1, Rpn4, Pdr3, and Yrr1. Biochem Biophys Res Commun 367: 249-255.

Teng X, Dayhoff-Brannigan M, Cheng WC, Gilbert CE, Sing CN, Diny NL, Wheelan SJ, Dunham MJ, Boeke JD, Pineda FJ, et al. 2013. Genome-wide consequences of deleting any single gene. Mol Cell 52: 485-494.

Treich I, Cairns BR, de los Santos T, Brewster E, Carlson M. 1995. SNF11, a new component of the yeast SNF-SWI complex that interacts with a conserved region of SNF2. Mol Cell Biol 15: 4240-4248.

Ververidis P, Davrazou F, Diallinas G, Georgakopoulos D, Kanellis AK, Panopoulos N. 2001. A novel putative reductase (Cpd1p) and the multidrug exporter Snq2p are involved in resistance to cercosporin and other singlet oxygen-generating photosensitizers in Saccharomyces cerevisiae. Curr Genet 39: $127-136$.

Wei W, McCusker JH, Hyman RW, Jones T, Ning Y, Cao Z, Gu Z, Bruno D, Miranda M, Nguyen M, et al. 2007. Genome sequencing and comparative analysis of Saccharomyces cerevisiae strain YJM789. Proc Natl Acad Sci 104: 12825-12830.

$\mathrm{Xu} \mathrm{Z,} \mathrm{Wei} \mathrm{W,} \mathrm{Gagneur} \mathrm{J,} \mathrm{Perocchi} \mathrm{F,} \mathrm{Clauder-Munster} \mathrm{S,}$ Camblong J, Guffanti E, Stutz F, Huber W, Steinmetz LM. 2009. Bidirectional promoters generate pervasive transcription in yeast. Nature 457: 1033-1037.

Zhang X, Cui Z, Miyakawa T, Moye-Rowley WS. 2001. Crosstalk between transcriptional regulators of multidrug resistance in Saccharomyces cerevisiae. J Biol Chem 276: 8812-8819.

Zheng W, Zhao H, Mancera E, Steinmetz LM, Snyder M. 2010. Genetic analysis of variation in transcription factor binding in yeast. Nature 464: 1187-1191.

Zhu C, Byers KJ, McCord RP, Shi Z, Berger MF, Newburger DE, Saulrieta K, Smith Z, Shah MV, Radhakrishnan M, et al. 2009. High-resolution DNA-binding specificity analysis of yeast transcription factors. Genome Res 19: 556-566. 


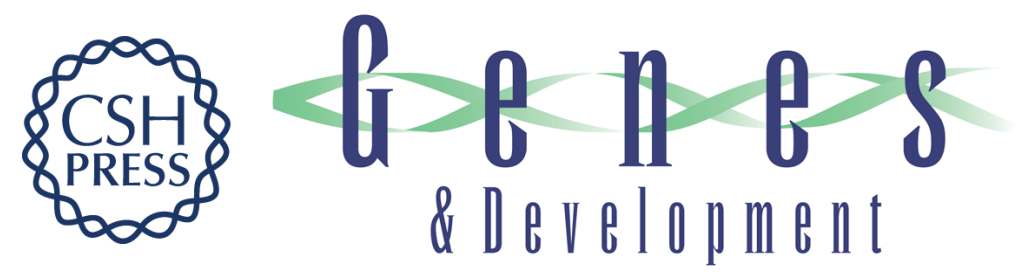

\section{Divergence in a master variator generates distinct phenotypes and transcriptional responses}

Jennifer E.G. Gallagher, Wei Zheng, Xiaoqing Rong, et al.

Genes Dev. 2014, 28:

Access the most recent version at doi:10.1101/gad.228940.113

\section{Supplemental http://genesdev.cshlp.org/content/suppl/2014/02/14/28.4.409.DC1 \\ Material}

References This article cites 48 articles, 21 of which can be accessed free at: http://genesdev.cshlp.org/content/28/4/409.full.html\#ref-list-1

Creative This article, published in Genes \& Development, is available under a Creative Commons Commons License (Attribution-NonCommercial 3.0 Unported), as described at License http://creativecommons.org/licenses/by-nc/3.0/.

Email Alerting Receive free email alerts when new articles cite this article - sign up in the box at the top Service right corner of the article or click here.

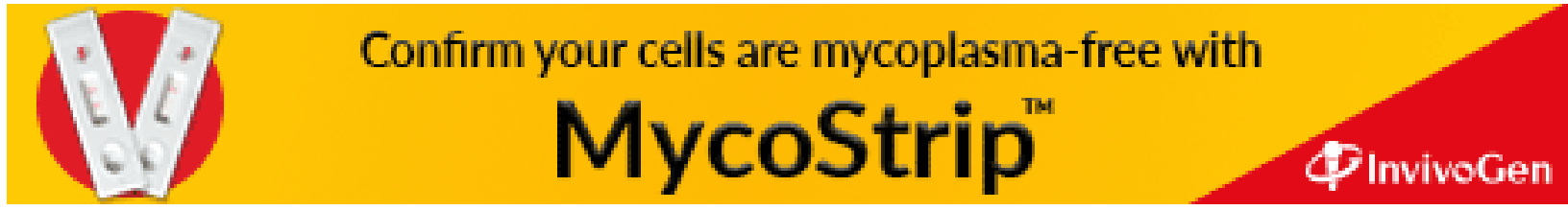

